\title{
Editorial
}

\section{Early Development and Epigenetic Programming of Body Functions in Birds}

Basic research on early ontogeny of body functions is carried out mainly in domestic bird species. Summarizing the results, it can be concluded that in birds the development of body functions but also of adaptation mechanisms related to changes in incubation environment starts early during the embryonic phase. However, during the final incubation period bird embryos, especially of precocial species, have well-developed physiological mechanisms, which enable them to react on environmental influences similar to the post-hatching period in a higher degree. Most functional systems of the organism develop from open loop systems without feedback control into closed control systems regulated by feedback mechanisms, which is a 'critical period' in the development of body functions. Some examples will be given as follows. Changes in incubation temperature, for instance, induce activation of thermoregulatory mechanisms [1]. After internal pipping, on increase or decrease in incubation temperature chicken and duck embryos are able to react with increase or decrease in blood flow of the chorioallantoic membrane, respectively. Respiratory rate is increasing with warming and distress call rate is increasing with cooling the eggs. Further, in Muscovy duck embryos the neuronal thermosensitivity of the thermoregulatory centre in the hypothalamus is similar with that of the post-hatching period [2, 3]. In chicken embryos the hypothalamic-pitatury-thyroidal axis is functional on embryonic day 19 [4], it means that feedback mechanisms are developed. The acoustic-sensory-cardiac axis in Muscovy ducks, for instance, is functional from embryonic day 27 and heart rate responses to acoustic stimulation are detectable [5]. In the domestic fowl circadian melatonin synthesis starts during embryonic life [6,7]. The amplitude of the rhythm increased considerably during the last two days of incubation, which indicates that melatonin may play an essential role in adapting the embryo to the postnatal environment [8].

Early environmental stimulation can improve the maturation of the respective physiological mechanisms ('training effect') and, if applied during 'critical periods' may have a long-lasting influence on the adaptability of the organism during the later life ('imprinting' of physiological control systems). In his classical studies on newly hatched goslings Konrad Lorenz analysed the development of social binding applying the term 'imprinting' to describe this process. One of his major ideas was that imprinting occurs during 'critical periods', which are limited and severely restricted to the animal's very early life [9]. In the course of the perinatal period, also 'imprinting' of physiological control systems occurs, which is a fundamental process of life (Fig. 1, [10]). During 'critical periods', the actual environment influences the development of the respective physiological control systems for the entire life period, especially induced by acquired changes in neural organization (e.g., in terms of synaptic plasticity) and expression pattern of related effector genes (functional/epigenetic perinatal programming). Suboptimal and altered environmental conditions may induce a malprogramming of respective functions, which could be the basis of 
diseases and metabolic or behavioural disorders during the entire lifetime. Maternal effects transferred to the embryo via hormone content in the egg may also induce epigenetic modifications of offspring phenotype [11]. In the canary, for instance, a high testosterone level in the yolk induces post hatching increased aggressive behaviour in female as well as male birds [12, 13].

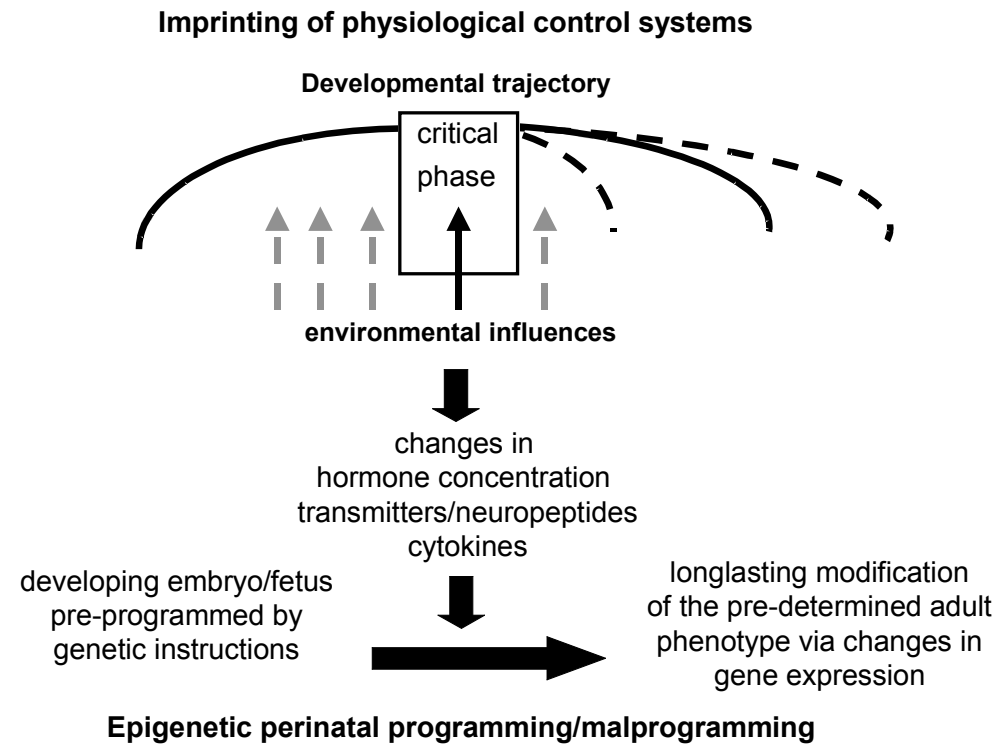

Fig. (1). Imprinting of physiological control systems by environmental factors during 'critical periods' of early development (modified from [10]). Günter Dörner developed the general concept of epigenetic perinatal programming of the lifetime function of fundamental regulatory systems. In this concept, hormones play a decisive role as environment-dependent organizers of the neuro-endocrineimmune system, which finally regulates all fundamental processes of life [14, 15]. During 'critical periods' hormones as well as neurotransmitters and cytotokines (as immune cell hormones) are involved in differentiation, maturation and functional programming of their own central nervous controllers within the respective physiological regulatory systems. They act as critical endogenous effectors, which transmit environmental informations (e.g. sensible or social input) to the genome. Finally, they are thereby also acting as epigenetic factors [16].

\section{Dr. Barbara Tzschentke (Guest Editor)}

Humboldt-University of Berlin Institute of Biology, Working Group Perinatal Adaptation, Philippstrasse 13

10115 Berlin,

Germany

E-mail: barbara.tzschentke@rz.hu-berlin.de

\section{REFERENCES}

[1] Tzschentke B. Attainment of thermoregulation as affected by environmental factors. Poult Sci 2007; 86: 1025-36.

[2] Tzschentke B, Basta D. Development of hypothalamic neuronal thermosensitivity in birds during the perinatal period. J Therm Biol 2000; 25: 119-23.

[3] Tzschentke B, Basta D, Janke O, Maier I. Characteristics of early development of body functions and epigenetic adaptation to the environment in poultry: focused on development of central nervous mechanisms. Avian Poult Biol Rev 2004; 15 (Suppl 3/4): 107-18.

[4] Muchow M, Bossis I, Porter TE. Ontogeny of pituitary thyrotrophs and regulation by endogenous thyroid hormone feedback in the chick embryo. J Endocrinol 2005; 184: 407-16. 
[5] Höchel J, Pirow R, Nichelmann M. Development of heart rate responses to acoustic stimuli in muscovy duck embryos. Comp Biochem Physiol 2002; 131A: 805-16.

[6] Zeman M, Gwinner E, Somogyiova E. Development of melatonin rhythm in the pineal gland and eyes of chick embryo. Experientia 1992; 48: 765-8.

[7] Lamosová D, Zeman M, Mackova M, Gwinner E. Development of rhythmic melatonin synthesis in cultured pineal glands and pineal cells isolated from chick embryo. Experientia 1995; 51: 970-5.

[8] Zeman M, Herichova I, Lamasová D, Gwinner E. Early development of circadian melatonin synthesis in adaptation of chickens. Proc. $11^{\text {th }}$ European poultry conference. Bremen; 2002: [published on CD].

[9] Lorenz K. Der Kumpan in der Umwelt des Vogels. J Ornithol 1935; 83: 137-213.

[10] Tzschentke B, Plagemann A. Imprinting and critical periods in early development. WPSJ 2006; 62: 626-37.

[11] Groothuis TGG, Schwabl H. Hormone-mediated maternal effects in birds: mechanisms matter but what do we know of them? Philos Trans R Soc B 2008; 363: 1647-61.

[12] Schwabl H. Maternal testosterone in avian egg enhances postnatal growth. Comp Biochem Physiol 1996; 114A: 271-6.

[13] Schwabl H. Maternal steroid hormones in the egg. In: Harvey S, Etches RJ, Eds. Perspectives in avian endocrinology. Bristol: Society for Endocrinol 1997; pp. 3-13.

[14] Dörner G. Perinatal hormone levels and brain organization. Anatom Neuroendocrinol 1975; 1: 245-52.

[15] Dörner G. Hormones and brain differentiation. Amsterdam: Elsevier 1976.

[16] Plagemann A. Perinatal nutrition and hormone-dependent programming of food intake. Horm Res 2006; 65(Suppl 3): 83-9. 